\title{
论 文
}

\section{连续面曝光陶瓷3D打印}

王赫 ${ }^{1}$, 兰红波 ${ }^{1}$, 钱垒 ${ }^{1}$, 赵佳伟 ${ }^{1}$, 许权 ${ }^{1}$, 朱晓阳 ${ }^{1}, 弓^{\circ}$ 广明 ${ }^{1}$, 鲁中良 ${ }^{2}$, 李涤尘 ${ }^{2}$

1. 青岛理工大学青岛市3D打印工程研究中心, 青岛 266033;

2. 西安交通大学机械制造系统工程国家重点实验室, 西安 710049

*E-mail: hblan99@126.com

收稿日期: 2018-10-07; 接受日期: 2018-12-14; 网络版发表日期: 2019-01-24

国家自然科学基金(批准号: 51775288)和山东省重点研发计划(编号: 2018GGX103022)资助项目

摘要 针对传统陶瓷3D打印存在打印效率低和成型件具有各向异性的不足和局限性, 提出一种连续面曝光陶瓷 $3 \mathrm{D}$ 打印新工艺，通过采用自主研发的复合富氧膜并结合配制的树脂基陶瓷浆料实现陶瓷素坏件的连续打印。阐 述连续面曝光陶瓷3D打印的基本原理和工艺流程; 研究陶瓷浆料的配制, 并利用搭建的实验平台揭示了关键工 艺参数对成形过程的影响和规律; 最后, 结合优化的工艺参数, 通过两个典型案例(镂空件和薄壁件)连续3D打印, 以及所打印的素坏件经脱脂烧结后性能的表征和测试, 证实了提出方法的可行性和有效性. 实现了陶瓷零件连续 $3 \mathrm{D}$ 打印工艺, 为探索高效、低成本连续陶瓷3D打印提供了一种全新的解决方案.

关键词陶瓷3D打印, 连续3D打印, 复合富氧膜, 陶瓷浆料, 增材制造

\section{1 引言}

陶瓷材料具有高强度、高硬度、耐高温、耐氧 化、耐腐蚀、化学性能稳定和轻质(低密度)等突出的 优点，在航空航天、生物医疗、汽车、电子、能源、 国防等诸多领域有着非常广泛的应用. 然而传统的陶 瓷成型技术面临加工困难(尤其是复杂几何形状结构 成型更为困难)、制造周期长、生产成本高等不足和 局限性, 制约陶瓷零件更广泛的应用. 近年来出现的陶 瓷3D打印技术为该问题提供了一种全新的解决方案, 陶瓷3D打印具有以下显著优势：(1) 无需原坏和模具, 生产周期短，制造成本低；(2) 制造精度高; (3) 可实现 几乎任意形状复杂结构成型，突破了传统工艺制造几 何形状的约束；（4）适合个性化定制和单件小批量生
产; (5) 打印材料种类广泛, 诸如氧化锆、氧化铝、磷 酸三钙、碳化硅、碳硅化钛、陶瓷前驱体、陶瓷基复 合材料等. 此外, 在微小零件 $3 \mathrm{D}$ 打印、陶瓷/金属复合 材料和功能梯度材料方面, 以及材料-结构-功能一体 化打印方面还具有独特的优势 ${ }^{[1 \sim 4]}$.

目前国内外学术界和产业界已经开发的陶瓷3D 打印工艺有十多种，主要包括：立体光固化成型 (SLA)、数字光处理(DLP)或者面曝光成型、选择性 激光烧结成型、黏结剂喷射成型、陶瓷熔化沉积成型 (fused deposition of ceramics, FDC)、叠层实体制造、 陶瓷墨水喷射打印成型、陶瓷浆料(挤出)直写成型 等 ${ }^{[4 ~ 9]}$. 近5年还出现一些商业化陶瓷3D打印设备和材 料提供商，例如奥地利Lithoz公司利用他们核心专利: 基于光刻的陶瓷制造(LCM，一种含有均匀分散的陶

引用格式: 王赫, 兰红波, 钱垒, 等. 连续面曝光陶瓷3D打印. 中国科学: 技术科学, 2019, 49: 681-689 Wang H, Lan H B, Qian L, et al. Continuous DLP ceramic 3D printing (in Chinese). Sci Sin Tech, 2019, 49: 681-689, doi: 10.1360/N092018-00338 
瓷粒子的感光树脂的选择性固化)工艺和开发的各种 陶瓷浆料 ${ }^{[10]}$, 已经推出商业化高精度陶瓷3D打印机 CeraFab 7500和8500. 2015年法国3DCeram 公司推出 首台工业级大幅面陶瓷3D打印机一CERAMAKER, 它 是一种立体光固化成型工艺，通过激光扫描混有树脂 的陶瓷材料, 实现光固化成型. 此外, 美国3D Systems 公司基于彩色喷印(CJP)开发出CeraJet陶瓷3D打印机; 美国ExOne公司等也相继推出黏结剂喷射陶瓷3D打 印机.

经过多年的发展，尽管陶瓷3D打印已经取得巨大 的进展和突破，但是现有的各种陶瓷3D打印技术仍然 面临以下挑战性难题: 打印效率低; 逐层打印致使成型 零件存在各向异性，导致后续烧结过程中易于出现裂 纹、变形等缺陷，严重影响打印件的质量、良率和精 度。2015年3月，美国Carbon3D公司Tumbleston等 人 $^{[11,12]}$ 在Science 上发表了一项颠覆性3D打印新技术: 连续液体界面生产技术(continuous liquid interface production，CLIP)，CLIP不仅极大地提高了3D打印速度, 同时还能够大幅提高打印精度和表面质量. 尤其是其 打破了 3D打印精度与速度不能同时提高(兼顾)的悖 论, 将3D打印速度提高100倍, 并且可以相对轻松地得 到无分层(layerless)的打印件. CLIP技术克服了高速连 续化3D打印的难题，在高速度、高精度、高表面质量 和高性能3D打印方面已经显示出独特优势(避免了传 统3D打印的台阶效应，克服传统3D打印制件各向异 性). 借鉴CLIP的成型原理, 本文创造性提出连续面成 型陶瓷3D打印新工艺，并从理论分析、实验工艺优化 和案例验证等方面开展了较为系统的研究，以期发展 和建立一种连续面曝光陶瓷3D打印新工艺.

\section{2 连续面曝光陶瓷3D打印基本原理和工艺 流程}

CLIP的打印材料是丙烯酸酯类的光敏树脂，其核 心功能模块成型窗口使用的是一种透UV光和透氧的 特氟龙薄膜. 不同于CLIP技术，连续面曝光陶瓷 $3 \mathrm{D}$ 打 印使用的材料陶瓷浆料是一种复合材料，具有很高的 黏度，并且含有固相成分、有机聚合物和无机材料混 合物，对于实现连续3D打印面临更大的挑战，现有的 CLIP技术无法满足连续陶瓷3D打印的技术要求(打印 材料、成型窗口、固化方式等). 为了实现连续打印对
于成型窗口和陶瓷浆料等必须采用全新的技术解决方 案，本文提出一种连续面曝光陶瓷3D打印新工艺，引 入一种复合富氧膜并结合特殊配制的树脂基陶瓷浆料 实现陶瓷素坏件的连续打印, 其成型基本原理如图1所 示. 整个打印系统主要包括的功能模块: 数字光处理模 块、储料槽、复合富氧膜、陶瓷浆料、成型零件、打 印平台等. 复合富氧膜是一种由多孔支撑层和富氧离 型层组成的双层复合薄膜，多孔支撑层采用含有微孔 的高透光PET膜，富氧离型层采用二甲基硅氧烷 (PDMS), 复合富氧膜不但具有高度透氧和透紫外光的 特性，而且具有优良的离型(低表面能)、抗拉伸、耐 候性等物化性能. 其成型原理是利用陶瓷浆料中的丙 烯酸光敏树脂的氧阻聚效应. 由于氧阻聚效应，进入 储料槽的氧气会抑制复合富氧膜顶部一定厚度内的陶 瓷浆料的固化反应，从而形成几十微米的“死区”(dead zone). 同时, 数字光处理模块发出的紫外光能固化“死 区”上方的陶瓷浆料. 由于已经固化的陶瓷部件与固定 在储料槽底壁复合富氧膜的离型膜PDMS没有发生黏 附, 所以打印时无需缓慢剥离, 从而能实现陶瓷零件的 连续打印成型.

基于CLIP的3D打印工艺的整个流程较为简单，而 连续面曝光陶瓷 $3 \mathrm{D}$ 打印的工艺流程则较为复杂, 涉及 的工艺步骤和工序较多, 完整工艺流程如图2所示, 主 要工艺步骤为: (1) 模型数据预处理. 首先进行模型缺 陷检查, 然后加支撑和模型分层, 最后将模型切片数据 编辑成连续投影图像传输到数字光处理模块; (2) 光敏 树脂基陶瓷浆料的配制. 将表面改性后的陶瓷粉和预 混液配制成陶瓷浆料(各组分确保均匀混合，避免陶瓷 材料团聚、光敏树脂与陶瓷材料密度不同导致分层); (3) 连续面曝光陶瓷 $3 \mathrm{D}$ 打印. 基本流程是 $Z$ 向工作台带 动成型平台浸入到陶瓷浆料中，使成型平台与成型窗 口的复合膜保持设定距离(“死区”的厚度)；Z向工作台 以设定的打印速度(优化出的工艺参数)连续提升，同 时数字光处理模块连续播放分层的数据信息，光源系 统产生的紫外光投射到陶瓷浆料上，成型平台在 $Z$ 向 工作台的提拉下将已固化部分从浆料中拉起，陶瓷浆 料持续填充已固化区域，整个过程连续无间断，直至 整个模型打印完成; (4) 从打印平台取下成型件, 将素 坏件进行清洗和烘干处理; (5) 脱脂和烧结. 根据陶瓷 浆料组分、固相含量的不同，采用优化出的脱脂烧结 工艺路线和参数(烧结温度、时间曲线), 首先脱脂, 然 


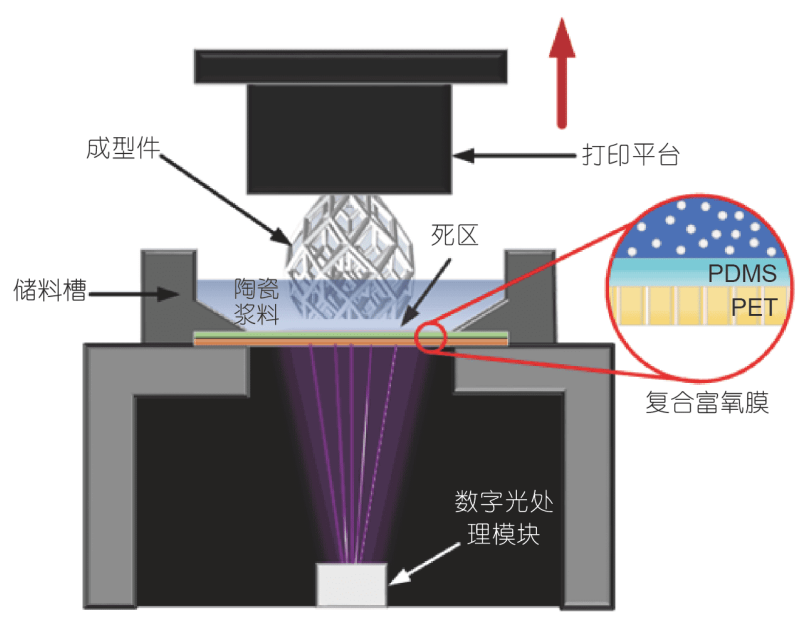

图 1 连续面曝光陶瓷3D打印原理

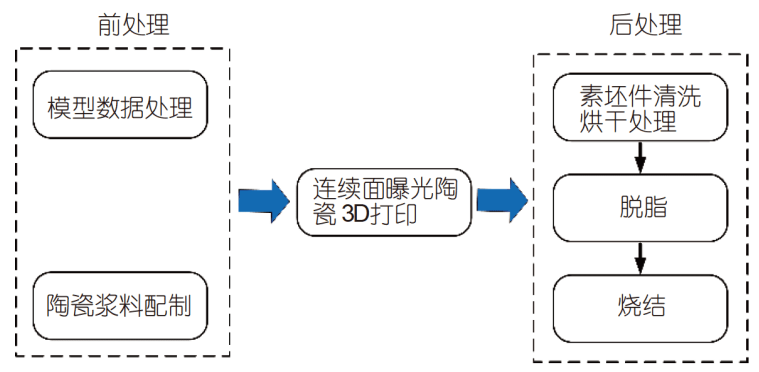

图 2 连续面曝光陶瓷制造工艺流程

后在烧结炉中进行烧结处理，完全去除陶瓷件中的有 机成分, 获得最终的陶瓷打印件. 其中模型数据处理 和陶瓷浆料配置属于打印前处理模块，清洗、脱脂、 烧结等属于打印后处理模块.

\section{3 实验装置、陶瓷浆料制备和打印工艺优 化}

\section{1 实验装置}

自主搭建的连续面曝光陶瓷3D打印实验装置如 图3所示，主要包括的功能模块有 $Z$ 向高精度位移台、 打印平台、储料槽、复合富氧膜、数字光处理模块 (储料槽的正下方)、控制模块等. Z 向高精度位移台采 用伺服电机+高精密丝杜(定位精度为 $10 \mu \mathrm{m}$ ); 数字光 处理模块采用高分辨率DLP光机，投影波段范围为 365 435 nm. 实验装置的核心功能模块是复合富氧膜,

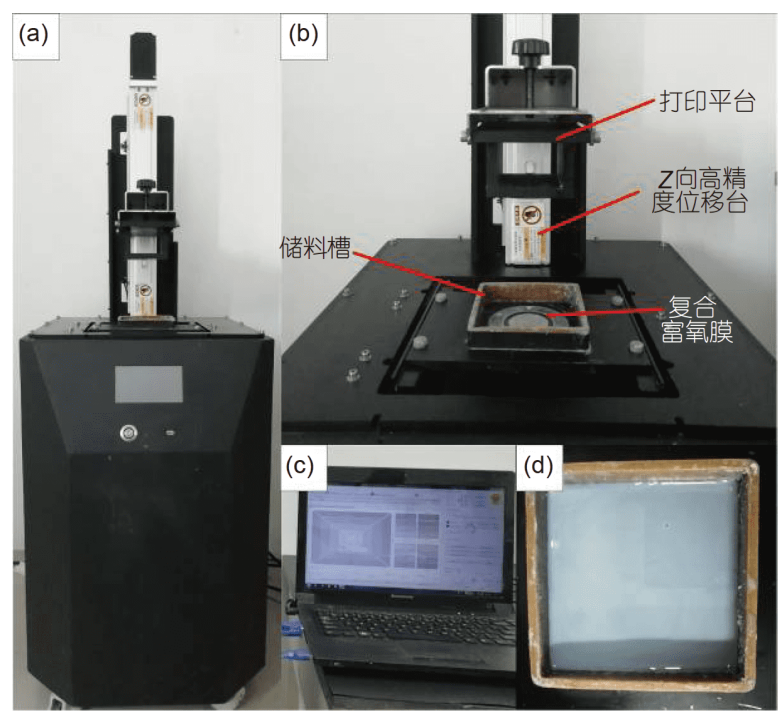

图 3 连续面曝光实验系统装置. (a) 连续面曝光实验系统 装置; (b) 实验系统装置主要结构; (c) 控制模块; (d) 储料槽

支撑层采用PET，离型膜采用PDMS，整个复合富氧膜 的最大成型面积为 $50 \mathrm{~mm} \times 50 \mathrm{~mm}$. 其中储料槽的尺 寸为 $150 \mathrm{~mm} \times 150 \mathrm{~mm}$.

\section{2 陶瓷浆料制备和性能表征}

陶瓷浆料对于连续面曝光陶瓷3D打印工艺实现 以及打印件质量、良率、精度和性能有决定性的影 响. 现有的各种陶瓷浆料不能满足连续面曝光陶瓷 $3 \mathrm{D}$ 打印的工艺要求，本文使用的陶瓷浆料为课题组自主 配制的. 主要制备过程如下.

（1）陶瓷粉表面改性. 用无水乙醇和硅烷偶联剂 KH-570配制 5\%浓度的混合溶液，再以体积比为 $5: 1$ 的 比例将平均粒径为 $5 \mu \mathrm{m}$ 的氧化锆陶瓷粉加入该混合 溶液，在 $45^{\circ} \mathrm{C}$ 的温度下磁力搅拌 $8 \mathrm{~h}$ ，过滤干燥得到表 面改性氧化锆陶瓷粉.

（2）陶瓷浆料配制. 自由基型光敏预混液和PAA作 为原料配制有机混合物溶液. 采用该混合物作为溶剂, 向其中分批次少量加入表面改性氧化锆陶瓷粉，同时 辅以高速 $(2500 \mathrm{r} / \mathrm{min})$ 搅拌 $8 \mathrm{~h}$, 直至粉料分散均匀, 再 以低速 $(500 \mathrm{r} / \mathrm{min})$ 搅拌除泡 $2 \mathrm{~h}$. 最终获得固相含量高 达 $50 \mathrm{vol} \%$ 的树脂基氧化锆陶瓷浆料。

陶瓷浆料性能表征: 经过测量, 所制备陶瓷浆料主 要工艺参数: 黏度 $427.8 \mathrm{mPa} \cdot \mathrm{s}$, 透射深度 $21.34 \mu \mathrm{m}$ ，临 界曝光强度 $20.71 \mathrm{MJ} / \mathrm{cm}^{2}$. 


\section{3 实验研究和工艺优化}

3.3.1 陶瓷浆料固相含量对打印过程的影响和规律

陶瓷浆料的固相含量直接决定陶瓷件是否能烧结 成功以及影响最终陶瓷件的致密度. 同时对于连续面 曝光陶瓷3 $\mathrm{D}$ 打印，固相含量直接影响着陶瓷浆料的黏 度和流动性(决定其对已固化区域的填充效率)，进而 影响整个打印过程和打印速度.

连续面曝光陶瓷3D打印对陶瓷浆料的基本要求： 低黏度(流变性能影响成型效率)和较高固相含量(影响 制件的孔隙率和性能). 但是这两个参数难以同时满 足，较高的固相含量会增大陶瓷浆料黏度，流动性变 差, 较低的固相含量打印要求的流动性能好, 但是打 印件的孔隙率高、表面质量和机械性能差. 因此，根 据实际的需求, 需要优化出合适的陶瓷浆料固相含量. 此外, 相比较CLIP和光固化成型使用的光敏树脂, 本 文使用的陶瓷浆料黏度较高，为保证打印质量和打印 效率，选择采用较低的打印速度，打印环境温度为 $25^{\circ} \mathrm{C}$. 所制备的陶瓷浆料的固相含量分成 6 个梯度范 围，最高测试固相含量为60 vol\%. 因为树脂中固体含 量的极限约为 $60 \%$, 超过该数值, 额外的陶瓷表现为陶 瓷粉末松散地黏附在烧结物上, 因此, 目前课题组没有 进行更高固相含量的陶瓷浆料的打印。其中本实验系 统打印的树脂件为对照实验件.

图4是陶瓷浆料固相含量与动力黏度、打印成功 率的关系，结果显示，随着陶瓷浆料的固相含量增大， 动力黏度也逐渐增大，而在对应固相含量下的打印成 功率却随之减小, 尤其是在接近 $60 \mathrm{vol} \%$ 时成型件的表 面出现了沟壑现象，这种现象与传统光固化的台阶效 应类似，原因是陶瓷浆料的黏度过高，在对已固化部

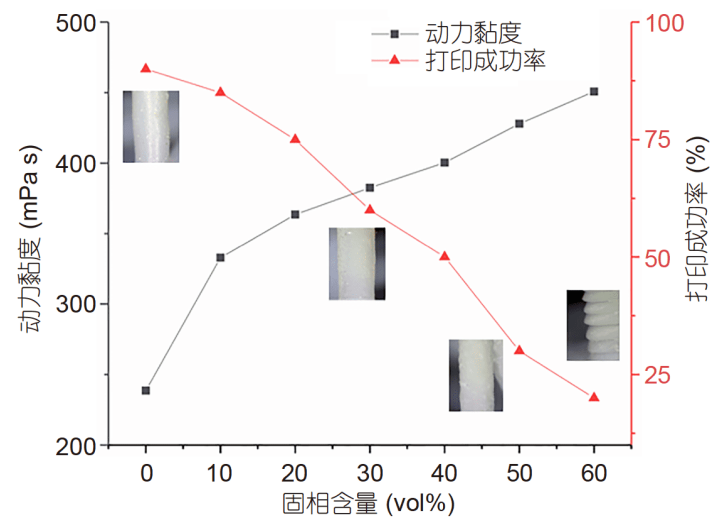

图 4 陶瓷浆料固相含量与动力黏度、打印成功率的关系
分空出区域未能完全填充造成的. 因此，随着陶瓷浆 料固相含量的增加，综合考虑打印成功率和随后脱脂 烧结成功率(开裂等缺陷)，本文所配制陶瓷浆料的固 相含量区间设定在 $30 \mathrm{vol} \% \sim 50 \mathrm{vol} \%$.

\subsection{2 复合富氧膜关键工艺参数对于成型过程的影响} 和规律

连续面曝光陶瓷3D打印成型窗口的基本要求: (1) 在确保富氧条件下同时具有高透紫外光性能; (2) 具有 优良的离型(低表面能)、抗拉伸、耐候性等物化性能. 课题组提出并研制了一种全新的复合富氧膜，它是一 种由多孔支撑层和富氧离型层组成的双层复合薄膜, 多孔支撑层采用含有微孔的高透光PET，富氧离型层 采用二甲基硅氧烷(PDMS). 其基本的制作流程是以聚 对苯二甲酸乙二醇酯(PET)微孔膜作为祄底, 将聚二甲 基硅氧烷(PDMS)均匀旋涂于表面, 并对整个复合富氧 膜进行加热固化. 该复合富氧膜具有制作简单、成本 低、离型效果优良、使用寿命长等优点，它能确保在 增加“死区”的透氧量的同时还具有较高的透光率，完 全满足连续面曝光陶瓷3 $\mathrm{D}$ 打印的成型窗口的性能 要求.

按照上述工艺制作的复合富氧膜截面如图5(a)所 示, 其中PDMS涂层是以旋涂的方法附着于PET层上最 终形成复合富氧膜. 通过多次实验，得到旋涂速度与 PDMS涂层厚度之间的关系如图5(b)所示, 结果显示当 旋涂速度低于 $1500 \mathrm{r} / \mathrm{min}$ 时, 随着旋涂速度的增大涂覆 的PDMS 厚度快速地减小; 但是当旋涂速度超过 $1500 \mathrm{r} / \mathrm{min}$, 涂覆的PDMS厚度变化较小. PDMS涂层的 厚度相对PET膜的厚度较大, 因此离型层PDMS的厚度 对复合富氧膜透光率影响较大, 需要优化出合适的 PDMS厚度. 如果离型层PDMS厚度小，尽管复合富氧 膜透光率较高, 但是会降低离型性能和使用寿命; 过厚 的PDMS涂层会影响入射光的折射率和复合膜的透光 率, 影响打印效率和精度. 因此我们制备了不同PDMS 厚度的复合富氧膜并用于实际打印和测试，研究结果 显示, 当PDMS涂层厚度为 $30 \sim 50 \mu \mathrm{m}$, 综合打印效果和 富氧性能较好. 因此, 根据实验优化的结果, 本文确定 的复合富氧膜的离型层PDMS厚度范围为30 50 $\mu \mathrm{m}$, 所使用的旋涂速度范围为1500 2000 r/min.

多孔支撑层PET的平均孔径对复合富氧膜的透氧 性有决定性影响. PET膜的孔径越大其透氧速率越高, 
透过PDMS 富氧层进入陶瓷浆料的氧气含量就越高, 所形成的对应“死区”的厚度越大．对于树脂连续面曝 光技术，改变“死区”厚度，可以通过调整打印初始位 置来保证其打印精度，然而对于连续面曝光陶瓷3D打 印, 存在陶瓷粉对入射光线的散射现象, 并且这种散射 现象对于陶瓷浆料是无法避免的，因此在陶瓷浆料固 相含量一定的情况下，散射现象的影响越小就意味着 打印精度越高. PET膜孔径大小对成型精度的影响如 图6，其中，图6(a)为不同孔径下对打印精度影响的示 意图. 对于相同厚度的离型层, 多孔支撑层PET的平均 孔径越大，其透氧量越高，进而导致“死区”厚度的增 加，更多的陶瓷粉使得投影入射光线的散射现象更加 严重，导致实际固化区域与理论固化区域的差值增大， 降低了整体的打印精度. 分别对平均孔径为 $0.22 \mu \mathrm{m}$ 和
$0.45 \mu \mathrm{m}$ 的PET膜进行实验测试, 采用 $35 \mathrm{vol} \%$ 固相含量 的陶瓷浆料, 打印速度设置为 $80 \mathrm{~mm} / \mathrm{h}$, 实验结果与分 析相符, 如图6(b)所示. 因此, 平均孔径为 $0.22 \mu \mathrm{m}$ 的 PET多孔膜更适合作为复合富氧膜的支撑层，可确保 素坏件的整体打印精度.

\subsection{3 打印速度对成型过程的影响和规律}

相比于其他陶瓷3D打印技术, 连续面曝光陶瓷3D 打印工艺的打印速度有着明显优势. 不过受限于本系 统数字光处理模块的光照强度和陶瓷浆料的性能，打 印速度理论上存在一个最优区间. 利用以上优化的工 艺参数, 并采用相同模型数据对整体的打印速度进行 测试，确定最优打印速度范围. 多次实验优化出的打 印速度范围为60 100 mm/h. 分别选取阈值范围的三
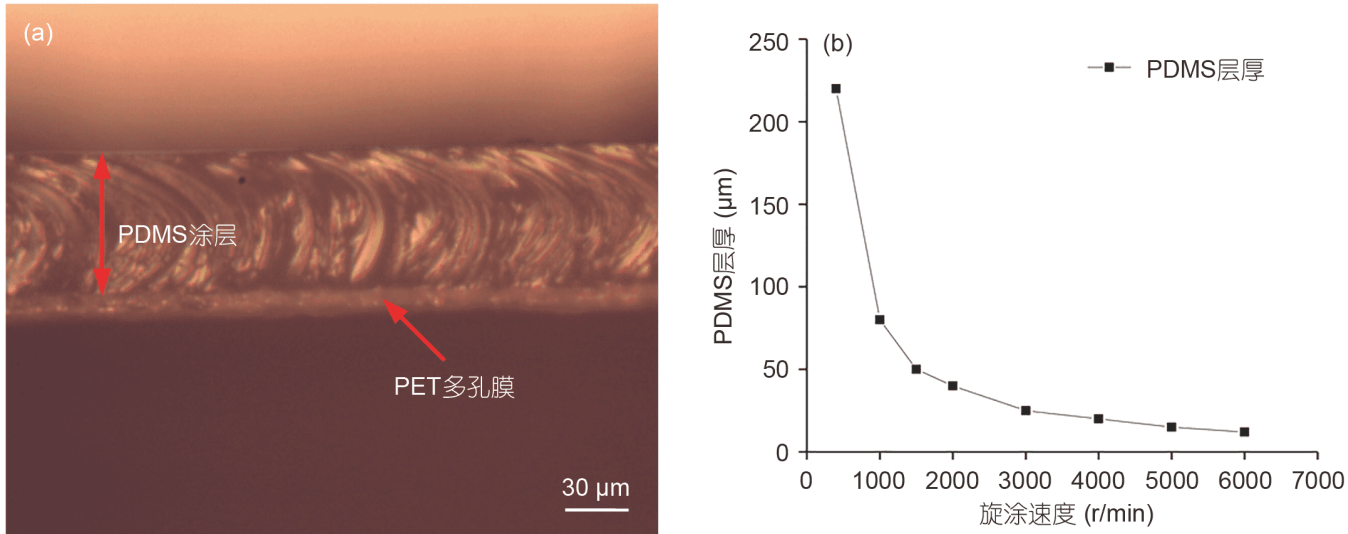

图 5 旋涂速度对复合富氧膜膜厚的影响. (a) 复合富氧膜的截面结构; (b) 旋涂速度与PDMS层厚的关系
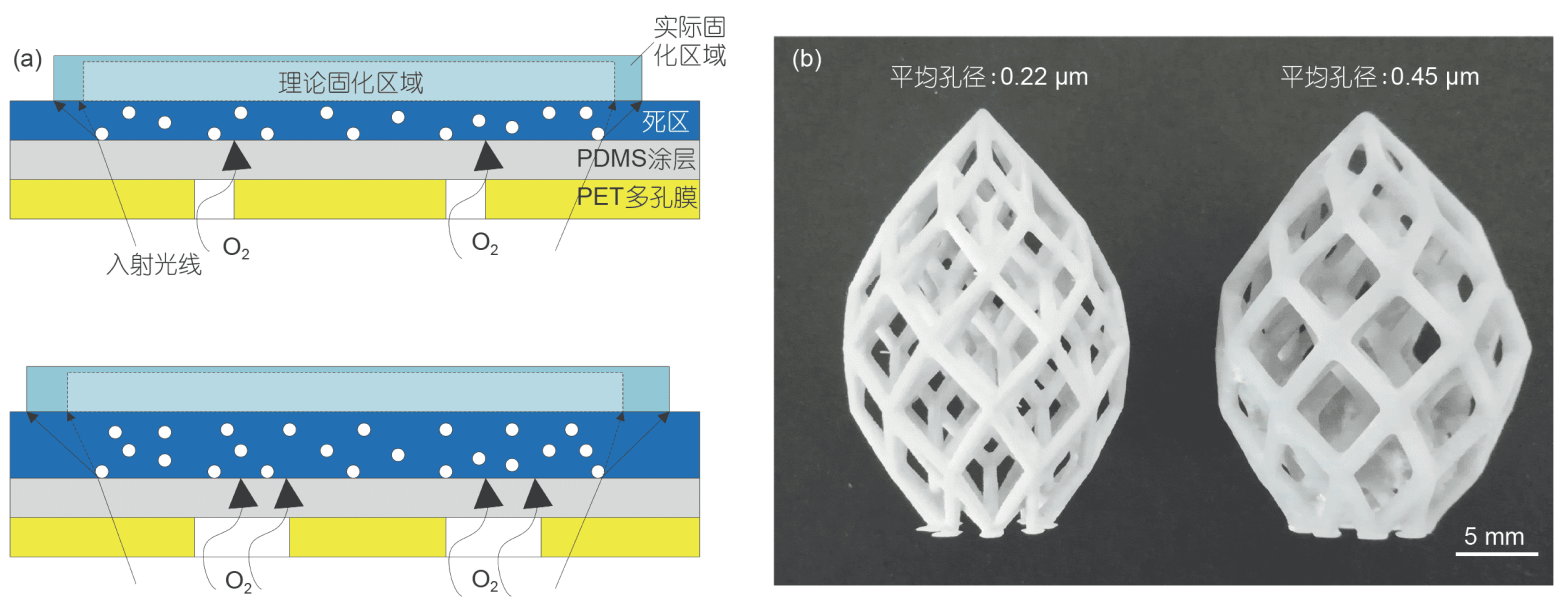

图 6 PET膜孔径大小对成型精度的影响. (a) 不同孔径下对打印精度影响的示意图; (b) 不同孔径下实际成型素坏件 
个典型速度进行打印，打印结果如图7所示. 当采用 $50 \mathrm{~mm} / \mathrm{h}$ 的打印速度时, 所打印的成型件精度较差, 这 是由于在较低速度下, 陶瓷粉对入射光线的散射较大, 增大了横向的成型尺寸. 当采用 $150 \mathrm{~mm} / \mathrm{h}$ 的打印速度 时, 成型件整体成型且呈现不均匀性, 甚至出现如图7 所示的结构缺失等重大缺陷，主要是由于陶瓷浆料的 低流动性所导致的对已固化区域的填充不均匀及部分 区域的填充缺失. 在最优打印速度范围内, 当采用 $100 \mathrm{~mm} / \mathrm{h}$ 的打印速度时，打印的零件成型精度较高， 结构完整且一致性好.

\section{4 案例研究}

利用本文提出的连续面曝光陶瓷3D打印技术，并 结合优化工艺参数, 以下通过两个典型打印案例, 展示 所提出方法的可行性和有效性.

\section{1 镂空陶瓷件}

选择镂空型零件作为第一个代表性案例. 以树脂 基氧化锆陶瓷浆料作为打印材料, 氧化锆陶瓷浆料固 相含量为 $50 \mathrm{vol} \%$, 其中陶瓷粉的平均粒径为 $5 \mu \mathrm{m}$. 成 型窗口复合富氧膜的支撑层采用平均孔径为 $0.22 \mu \mathrm{m}$ 且厚度为 $10 \mu \mathrm{m}$ 的PET微孔膜, 离型层为厚度为 $50 \mu \mathrm{m}$ 的PDMS. 打印速度设为 $80 \mathrm{~mm} / \mathrm{h}$. 连续面曝光陶瓷 $3 \mathrm{D}$ 打印制造的素坏件经过脱脂烧结后得到的陶瓷零件如 图8(a)所示. 实验打印结果显示: 制件没有开裂和裂纹 等缺陷，表面质量和尺寸精度高，体积收缩率约为 $24 \%$. 通过显微放大图可以看出表面连续性较好, 即未 出现台阶现象, 也未出现表面裂纹和较大孔隙.

\section{2 薄壁陶瓷件}

薄壁陶瓷件的制造是陶瓷3D打印的难点，第二个 案例选择薄壁陶瓷件连续3D打印，验证本系统的打印 效果和性能. 成型窗口复合富氧膜参数与上述案例相 同，采用的氧化锆陶瓷浆料固相含量为 $40 \mathrm{vol} \%$ ，打印 速度设为 $100 \mathrm{~mm} / \mathrm{h}$. 降低陶瓷浆料的固相含量有助于 增加其流动性, 提高填充速度.

首先打印了一些结构较为简单的中空薄壁件，并 进行了脱脂和烧结，如图9(a)所示，成型效果较好，未 出现瑕疵. 在此基础上又进行了结构较为复杂的小型 叶轮的打印，打印的素坏件和脱脂烧结后得到对应陶

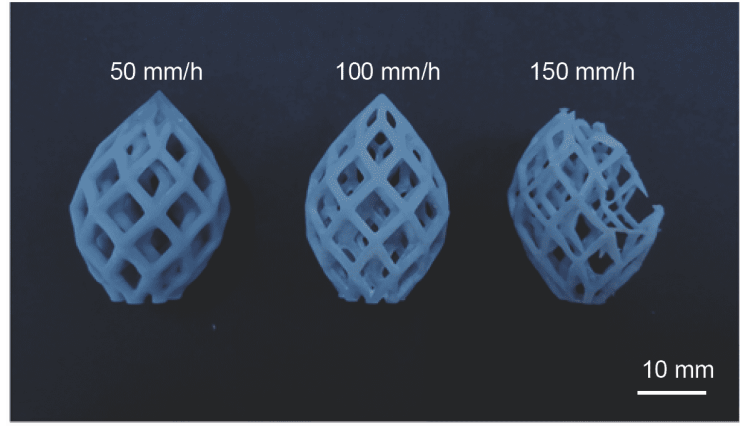

图 7 不同打印速度下的成型情况对比
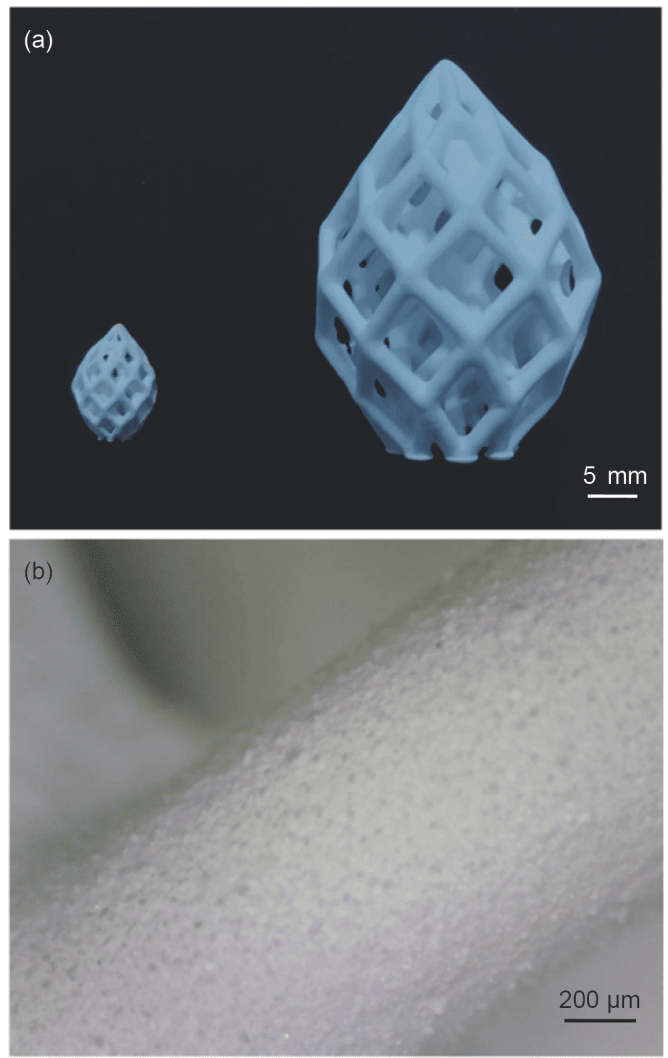

图 8 基于连续面曝光陶瓷3D打印制造的镂空陶瓷零件(脱 脂烧结后). (a) 两种不同镂空零件; (b) 镂空零件局部放大图

瓷件，如图9(b)所示. 实验结果显示: 所制造的陶瓷零 件表面质量好, 没有出现开裂和裂纹等缺陷.

\section{5 陶瓷件性能检测和结构表征}

\section{1 脱模烧结后陶瓷零件表观密度和显气孔率}

采用阿基米德原理对烧结后的陶瓷零件的表观密 度和显气孔率进行检测，检测样本为打印的氧化锆圆 

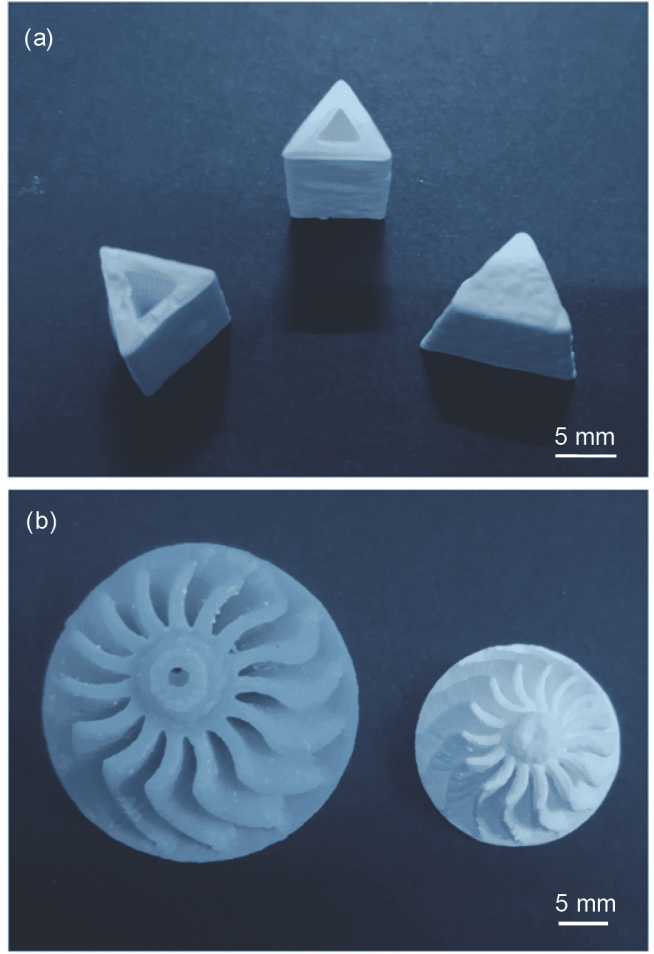

图 9 基于连续面曝光陶瓷3D打印制造的薄壁零件. (a) 简 单薄壁结构的陶瓷件; (b) 小型叶轮的素坏件和陶瓷件

柱形样件, 参考标准为GB/T25995-2010, 样本浸入采 用煮沸法 ${ }^{[13]}$, 具体计算公式如下:

表观密度为

$\rho=m_{1} /\left(m_{1}-m_{2}\right)-\rho_{1}$

显气孔率为

$\pi=\left(m_{3}-m_{1}\right) /\left(m_{3}-m_{2}\right) \times 100 \%$,

式中, $m_{1}$ 为陶瓷样件完全干燥时的质量, $m_{2}$ 为陶瓷样 件在蒸馏水中的浮重, $m_{3}$ 为陶瓷样件完全浸入水中的 质量, $\rho_{1}$ 为所用蒸馏水的密度.

通过高精度的电子天平测得陶瓷样件完全干燥时 的质量 $m_{1}$ 为 $4.325 \mathrm{~g}$, 浮重 $m_{2}$ 为 $3.617 \mathrm{~g}$, 浸入蒸馏水中 的质量 $m_{3}$ 为 $4.624 \mathrm{~g}$, 蒸馏水的密度为 $0.999 \mathrm{~g} / \mathrm{cm}^{3}$, 由 公式(1)计算的表观密度为 $5.109 \mathrm{~g} / \mathrm{cm}^{3}$, 为氧化锆粉体 理论密度 $5.89 \mathrm{~g} / \mathrm{cm}^{3}$ 的 $86.7 \%$, 由公式(2)计算的显气孔 率为 $29.79 \%$.

\section{2 打印陶瓷件的微观结构表征和成分分析}

如上述案例研究中的成型陶瓷件所示，其表面都
较为良好, 未出现明显的瑕疪. 为了进一步地验证其 表面质量，对使用同样工艺参数和相同陶瓷浆料制造 的试样，采用场发射扫描电子显微镜 SEM(SU-8010, 日本)进行观测, 结果如图10所示. 图10(a)低倍放大图 中显示表面似乎有较小孔隙，但在进一步放大后可以 看出只是个别的表面坑洼, 这种现象出现的原因可能 是陶瓷浆料固相含量较低导致浆料分布不均匀，而在 脱脂烧结后出现微小坑洼. 图10(b)高倍放大图中可以 看出陶瓷粉经烧结后都已黏结在一起，但是表面形貌 较为粗粘，需要在以后的设计制造中加入一些更加有 效的辅助后处理工艺.

陶瓷粉、素坏件和陶瓷零件则均采用 $\mathrm{X}$ 射线衍射 仪(EXPLORER，北京科曼科技有限公司)对其进行化 学成分和材料结构的检测. 图11为本实验所使用的氧 化锆陶瓷粉末、陶瓷素坏和烧结后陶瓷粉的综合 XRD图谱. 整个制造工艺过程分为陶瓷浆料的制作、 陶瓷素坏件的打印和陶瓷件的脱脂烧结. 由于脱脂温 度在 $400^{\circ} \mathrm{C}$ 以下，因此陶瓷粉体和陶瓷素坏的主要成 分为单斜相氧化锆 $\left(m-\mathrm{ZrO}_{2}\right)$. 整个烧结过程的温度范 围为 $400 \sim 1450^{\circ} \mathrm{C}$, 且升温过程中单斜相氧化锆向四方
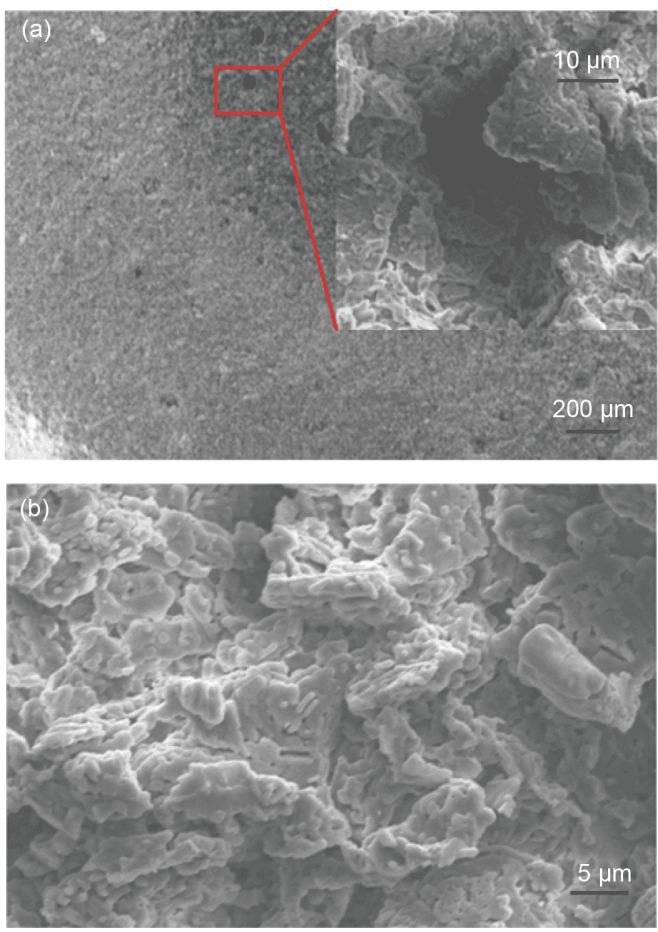

图 10 烧结后陶瓷件外表面SEM图. (a) 低倍 $(\times 40$ 倍); (b) 高 倍(×2000倍) 


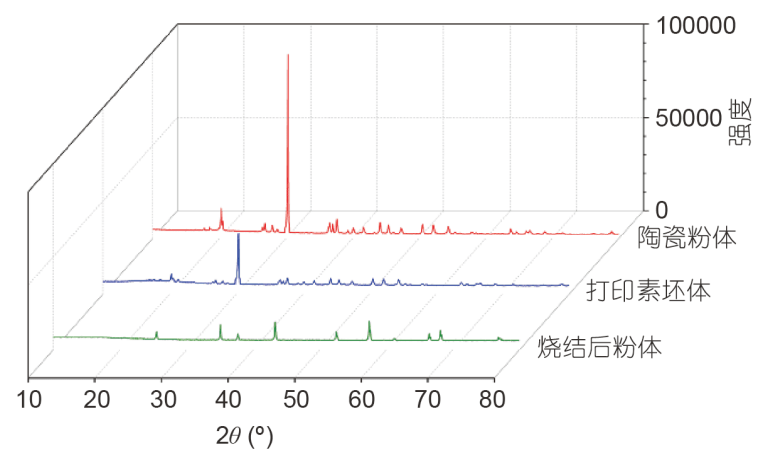

图 11 氧化锆陶瓷粉体、打印素坏和烧结后陶瓷粉末的 XRD图

相氧化锆 $\left(t-\mathrm{ZrO}_{2}\right)$ 的转变温度为 $1200^{\circ} \mathrm{C}$, 体积收缩; 降 温过程中四方相氧化锆向单斜相转变温度为 $1000^{\circ} \mathrm{C}$, 体积膨胀. 从图11中可以看出: 烧结后的陶瓷粉在经过 相变后的体积小于未烧结的陶瓷粉，因此脱脂烧结后 的陶瓷件主要成分为四方相氧化锆. 加入氧化钇等稳 定剂的四方相氧化锆具有高强度韧性和耐磨性，因此 在医疗用陶瓷刀具和汽车发动机针阀等领域具有广泛 的应用前景，但还需要进一步地优化稳定剂和陶瓷粉 的配比以及烧结工艺路线.

\section{6 结论}

本文提出一种连续面曝光陶瓷3D打印新工艺，并 从理论分析、实验工艺优化、典型样件打印、制件性 能测试和表征等方面开展了系统的研究，得到以下重 要结论.

（1）通过引入一种复合富氧膜并结合研制的树脂
基陶瓷浆料，实现陶瓷素坏件连续打印。其成型原理 是利用陶瓷浆料中的丙烯酸光敏树脂的氧阻聚效应, 由于氧阻聚效应，进入储料槽的氧气会抑制靠近复合 富氧膜底部的陶瓷浆料树脂发生固化，形成几十微米 的“死区”. 同时，数字光处理模块发出的紫外光能固 化“死区”上方的陶瓷浆料. 由于已经固化的陶瓷件与 固定在储料槽底壁复合富氧膜的离型膜PDMS没有发 生黏附, 所以打印时无需缓慢剥离, 从而能实现陶瓷零 件的连续打印成型.

(2) 提出的复合富氧膜是由多孔支撑层和富氧离 型层组成的双层复合薄膜, 多孔支撑层采用含有微孔 的高透光PET, 富氧离型层采用二甲基硅氧烷(PDMS), 复合富氧膜不但具有高度透氧和透紫外光的特性，而 且具有优良的离型(低表面能)、抗拉伸、耐候性等物 化性能.

（3）实验优化的主要工艺参数: 树脂基陶瓷浆料的 固相含量应控制在 $30 \mathrm{vol} \%$ ～ $0 \mathrm{vol} \%$; 复合富氧膜离 型层PDMS的厚度为 $50 \mu \mathrm{m}$; 多孔支撑层选用平均孔径 为 $0.22 \mu \mathrm{m}$ 的PET微孔膜; 最优打印速度范围在 60 $\sim 100 \mathrm{~mm} / \mathrm{h}$.

(4) 通过优化的工艺参数, 实现了镂空陶瓷件和小 叶轮等薄壁陶瓷件的连续3D打印，经脱脂烧结后，性 能和表面质量良好; 样件经测试，表观密度为5.109 $\mathrm{g} / \mathrm{cm}^{3}$, 显气孔率为 $29.79 \%$.

本文提出的连续面曝光陶瓷 $3 \mathrm{D}$ 打印工艺为陶瓷 3D打印提供了一种全新的解决方案，后续对于成型机 理和规律、陶瓷浆料(进一步提高固相含量)优化、打 印速度提高、复合富氧膜性能改进、复杂高致密度零 件制造等问题, 还需开展更为深入的研究.

\section{参考文献}

1 Eckel Z C, Zhou C, Martin J H, et al. Additive manufacturing of polymer-derived ceramics. Science, 2016, 351: 58-62

2 Hwa L C, Rajoo S, Noor A M, et al. Recent advances in 3D printing of porous ceramics: A review. Curr Opin Solid State Mater Sci, 2017, 21: 323-347

3 Gualtieri T, Bandyopadhyay A. Additive manufacturing of compositionally gradient metal-ceramic structures: Stainless steel to vanadium carbide. Mater Design, 2018, 139: 419-428

4 Chartier T, Dupas C, Lasgorceix M, et al. Additive manufacturing to produce complex 3D ceramic parts. J Ceram Sci Technol, 2014, 6: 95-104

5 Costa E, Duarte J P, Bártolo P. A review of additive manufacturing for ceramic production. Rapid Prototyping J, 2017, 23: 954-963

6 Yun J S, Park T W, Jeong Y H, et al. Development of ceramic-reinforced photopolymers for SLA 3D printing technology. Appl Phys A, 2016, 122: 629

7 Deckers J, Vleugels J, Kruth J P. Additive manufacturing of ceramics: A review. J Ceram Sci Technol, 2014, 5: 245-260 
8 Scheithauer U, Slawik T, Schwarzer E, et al. Additive manufacturing of metal-ceramic-composites by thermoplastic 3D-printing (3DTP). J Ceram Sci Technol, 2015, 6: 125-131

9 Huang B, Bártolo P J. Rheological characterization of polymer/ceramic blends for 3D printing of bone scaffolds. Polymer Testing, 2018, 68: 365378

10 Scheithauer U, Schwarzer E, Moritz T, et al. Additive manufacturing of ceramic heat exchanger: Opportunities and limits of the lithographybased ceramic manufacturing (LCM). J Materi Eng Perform, 2018, 27: 14-20

11 兰红波, 李涤尘, 卢秉恒. 微纳尺度3D打印. 中国科学: 技术科学, 2015, 45: 919-940

12 Tumbleston J R, Shirvanyants D, Ermoshkin N, et al. Continuous liquid interface production of 3D objects. Science, 2015, 347: 1349-1352

13 杨飞, 连芩, 武向权, 等. 陶瓷面曝光快速成型工艺研究. 机械工程学报, 2017, 53: 138-144

\title{
Continuous DLP ceramic 3D printing
}

\author{
WANG He ${ }^{1}$, LAN HongBo ${ }^{1}$, QIAN Lei ${ }^{1}$, ZHAO JiaWei ${ }^{1}$, XU Quan ${ }^{1}$, ZHU XiaoYang ${ }^{1}$, \\ ZHANG GuangMing ${ }^{1}$, LU ZhongLiang ${ }^{2} \&$ LI DiChen ${ }^{2}$ \\ ${ }^{1}$ Qingdao Engineering Research Center for 3D Printing, Qingdao Technological University, Qingdao 266033, China; \\ ${ }^{2}$ State Key Laboratory for Manufacturing System Engineering, Xi'an Jiaotong University, Xi'an 710049, China
}

In order to resolve the problems of the low efficiency and the anisotropy of printed object in the traditional ceramic 3D printing techniques, a novel continuous digital light processing (DLP) ceramic 3D printing implemented by utilizing the combination of an oxygen-rich composite thin film and the resin-based ceramics slurry has been proposed and developed. The basic principle and the process flow of the proposed continuous ceramic 3D printing are described. Then, the ceramic slurry used for this proposed 3D printing technique is investigated and optimized, and the effects and the rules of primary process parameters on forming process are revealed by conducting a series of experiments. Finally, two typical cases are provided to demonstrate the feasibility and validity of the proposed process. As a result, a continuous DLP ceramic 3D printing has been fulfilled, which provides a promising and novel solution for high-efficiency, low-cost ceramic 3D printing.

ceramic 3D printing, continuous 3D printing, oxygen-rich composite film, ceramic slurry, additive manufacturing doi: $10.1360 /$ N092018-00338 